\title{
HOT-ATOM CHEMISTRY
}

\author{
A.A. GORDUS \\ Department of Chemistry, The University of Michigan, \\ Ann Arbor, 48109 (USA)
}

(Received January 10, 1990)

\begin{abstract}
Research in the chemical effects of the nuclear transformations during the past 50 years is reviewed.
\end{abstract}

\section{Introduction}

Hot-atom chemistry (HAC) is the study of the chemical reactions that occur between high-energy atoms and ions and (usually) thermal-energy atoms, molecules, ions, and radicals. Studies involve atoms and ions that are produced photochemically, by velocity selection as in atomic-beam studies, as well as by nuclear processes. This review of a half-century of hot-atom chemistry is concerned only with the last means of producton and a more appropriate title would be the Chemical Effects of Nuclear Transformations (CENT). In fact, the two phrases, HAC and CENT are often used interchangeably in describing the chemical processes that occur when hot atoms. and ions are produced by nuclear means.

SZILARD and CHALMERS ${ }^{1}$ in 1934 were the first to report a study of CENT. They showed that when ethyl iodine was irradiated with neutrons, much of the ${ }^{128} \mathrm{I}$ produced in the ${ }^{127} \mathrm{I}(\mathrm{n}, \gamma){ }^{128} \mathrm{I}$ nuclear reaction could be extracted by water, indicating that the ${ }^{128} \mathrm{I}$ ruptured from its parent compound as a result of the nuclear process. Very few studies were reported during the next 10-15 years, primarily because of the lack of availability of neutron sources. As research nuclear reactors with high neutron fluxes became available in the late 1940's and early 1950's, interest in CENT studies increased markedly and attracted the interest of researchers worldwide. Over two thousand articles have been published dealing with all aspects of CENT and this review of a half-century of hot atom chemistry provides only a sampling of the work in this field.

A large amount of energy is released a nuclear transformation and a fraction of this energy can be imparted as recoil energy to the atom (isotope) undergoing the nuclear transformation, causing the activated isotope to dissociate from the molecule 
to which it was bonded. Typical energies are about $200 \mathrm{eV}$ in the (n, $\gamma$ ) activation process, $192,000 \mathrm{eV}$ for the ${ }^{3} \mathrm{~T}$ in the ${ }^{3} \mathrm{He}(\mathrm{n}, \mathrm{p})^{3} \mathrm{~T}$ activation, and even larger energies in the production of isotopes by nuclear fission. A $2.0 \mathrm{MeV}$ beta decay can result in a recoil energy of up to about $20 \mathrm{eV}$. And even the relatively weak isomeric transition (IT) process can result indirectly in a recoil energy of a few $\mathrm{eV}$ since IT is often accompanied by the release of a large number of Auger electrons resulting in a distribution of positive charge on the molecule with subsequent Coulombic repulsion and dissociation of the IT-activated isotope.

Bond energies are typically less than about $4 \mathrm{eV}$ so that bond rupture is almost always assured for the more energetic activation processes. The hot atom usually cannot form a stable bond in the first few collisions with room-temperature $(0.025$ $\mathrm{eV})$ molecules. Only after sufficient collisions which involve the loss of much of the excess energy is the hot atom able to react to form a stable product. Complicating the picture is the fact that many if not most of the hot atoms are really not atoms when they are formed but more likely are ions in varying ionic excitation states. And, even though translational kinetic energy can be lost in sequential collisions, the ionic and excitation state of the "slowed down" hot atom may not be known.

Although atomic and molecular beam studies allow investigating individual chemical interactions at selected energies, hot atom chemical studies only allow observing the overall chemical processes. However, the judicious use of additives that can moderate the reaction by absorbing excess kinetic energy of the hot atoms (inert gases for instance) and/or neutralize charged species (additives with ionization potentials than are lower than that of the hot atom), has made it possible to sort out and describe some of the hot-atom chemical mechanisms.

Most of the early studies were concerned with the hot atom chemistry of $(n, \gamma)$ and IT-activated halogen isotopes and ${ }^{3} \mathrm{~T}$ activated by the ${ }^{3} \mathrm{He}(\mathrm{n}, \mathrm{p}){ }^{3} \mathrm{~T}$ process, in all three phases: solid, liquid, and gas. In later years interest expanded to include, among others, studies of the HAC of metal-organic and inorganic solid compounds, other methods of production such as beta decay, and the use of HAC to study nuclear fission.

Hot atom studies have relevance to many other chemical studies. Upper atmosphere chemistry where high-energy atoms are produced is just one example.2,3 Another is ion-implantation. ${ }^{4-7}$ Labeling of chemical compounds and radiopharmaceuticals is yet another example. . $^{71}$

A number of reviews ${ }^{12-15}$ and symposia ${ }^{16,17}$ on hot atom chemistry have appeared in recent years and detail the progress in this field. 


\section{Experimental methods}

Since the product isotope in HAC studies is radioactive, radiochemical separation methods are used to identify and quantify the products. The earliest studies depended on solvent extraction, column and chemical-separation methods. In the $1950 \mathrm{~s}$, radiogas chromatographic ${ }^{12,18}$ methods were developed and are now routinely used in most studies of gas-phase HAC. Radioion, thin layer, and paper chromatography, $12,19,20$ as well as electrophoresis and ion-exchange separation are usually used when working with solid samples or dissolved molecules that are not amenable to gas chromatographic separation. The Moessbauer effect has also been used to study HAC products in solids. ${ }^{21}$

\section{Theoretical models}

The activation process usually results in atoms/ions having a wide (and often unknown) distribution of initial energies ${ }^{22}$ following rupture from the molecule to which the atom was initially bound. However, partial cancellation of prompt gammaray momenta in $(n, \gamma)$ activation can result in what is usually a small but measurable degree of failure to bond-rupture ${ }^{23}$ unlike low-energy beta-decay activation, unaccompanied by gamma ray emission as in the case of ${ }^{14} \mathrm{C}$ and ${ }^{3} \mathrm{~T}$ decay, which can result in appreciable non-rupture. ${ }^{24}$

The earliest models of hot atom gas phase reaction kinetics were based on a billiard-ball model. ${ }^{25}$ ESTRUP and WOLFGANG ${ }^{26}$ developed a kinetic model for hot atom reactions similar to that used by WIGNER to describe neutron slowing down processes. ${ }^{27}$ More recent approaches have included contributions for collisional dissociation, 28 use of nonequilibrium time dependent theory, ${ }^{29-31}$ a steady-state theory, 32,33 and evaluation of potential energy surfaces. ${ }^{34}$ Nonequlibrium theory has also been applied to hot atom reactions in liquids and solids. ${ }^{35}$ Other models for liquids and solids are based on back-diffusion, ${ }^{36}$ an exploding lattice, ${ }^{37}$ and cage effects. ${ }^{12}$ Not all of the activated atoms react as hot atoms. Many eventually reach thermal energies and become stabilized in conventional chemical reactions, often by reaction with chemical scavengers. However, in some cases the thermalized atoms/ions may still be in excited electronic states permitting reaction by processes not normally occuring in simple room temperature gas-phase chemical reactions. ${ }^{38}$ 


\section{Halogen HAC-organic systems}

The HAC of all four halogen atoms: flourine, ${ }^{39-44}$ chlorine, ${ }^{45-51}$ bromine, ${ }^{52-59}$ and iodine, ${ }^{60-64}$ have been in organic systems in all three phases and comparise a major portion of the hot atom literature. A variety of activation processes have been used, including: $(\mathrm{n}, \gamma),(\gamma, \mathrm{n}),(\mathrm{n}, 2 \mathrm{n})$, as well as isomeric transition: (IT), especially for $\mathrm{Br}$, which has two stable isotopes that can be easily activated. Various hydrogen/deuterium and halogen isotope effects were also observed. ${ }^{65-68}$

It is not uncommon for a number of organic products to be formed in the HAC reactions with organic target molecules. For instance, irradiation of a gaseous mixture of $\mathrm{Br}_{2}$ and propane results in more than eight detectable organic bromide products. Different inert gases, mixed at various mole-fractions, provide information on how hot-atom thermalization processes effect the yield of each the products. Results are usually given in terms of the total "organic yield", which is the fraction of the activated radioactive halogen found in organic form, as well as in terms of the fraction of the radioactive halogen found in the various organic chemical forms.

\section{Halogen HAC-inorganic systems}

Early studies were concerned with the chemical fate of hot halogen atoms in alakli halides and oxyhalides. ${ }^{69-72}$ More recent research involved studies of other solids such as $\mathrm{NH}_{4} \mathrm{IO}_{3}$, mixed solids, aqueous solutions at various $\mathrm{pH}$, the effect of thermal annealing of the irradiated crystals, and reactions of hot halogen originating from inorganic salts soluble in organic solvents. ${ }^{73-80}$

\section{Other metal organic/inorganic solids}

Studies of the HAC of metal-containing solids is extensive and consist of more than 300 articles; two recent books ${ }^{12,14}$ summarize modern developments in the field. The majority of the studies of inorganic solids are concerned with the HAC of the first-row transition metals ${ }^{81-92}$ and in particular chromium, manganese, and cobalt compounds, with fewer studies of the second and third row transition metals. Studies of rare-earth compounds have received very little attention unlike the group IIIAVIA metal compounds, and especially those of mercury, tin, selenium, tellurium, cadmium, silicon, and arsenic. 


\section{Tritium HAC}

High-energy tritons can be generated in the gas phase by the ${ }^{3} \mathrm{He}(\mathrm{n}, \mathrm{p})^{3} \mathrm{~T}$ reaction and in condensed phases by the ${ }^{6} \mathrm{Li}(\mathrm{n}, \alpha)^{3} \mathrm{~T}$ reaction. As in conventional chemical studies, the HAC of hydrogen (in this case tritium) has also been studied extensively and a few hundred articles are devoted to studies of the HAC of tritium, mostly for gas-phase reaction systems. ${ }^{93}$ In addition, chemical studies of hot hydrogen or deuterium atoms produced by photodissociation of hydrogen or deuterium halides provide complimentary information. Because of the elctronic simplicity of hydrogen atoms, it has been much easier to postulate mechanisms. ${ }^{94-103}$ Reactions with a variety of target molecules and additives have been reported. ${ }^{104-115}$

\section{Carbon, nitrogen, phosphorus and sulfur HAC}

Studies of the HAC of these four elements are of particular interest because of their relevance to labelling of organic compounds and radiopharmaceuticals. Carbon11 can be generated by a variety of means including the ${ }^{14} \mathrm{~N}(\mathrm{p}, \alpha)^{11} \mathrm{C}$ and the ${ }^{12} \mathrm{C}(\mathrm{n}, 2 \mathrm{n}){ }^{11} \mathrm{C}$ processes whereas carbon-14 is usually produced by the ${ }^{14} \mathrm{~N}(\mathrm{n}, \mathrm{p})^{14} \mathrm{C}$ reaction. ${ }^{116-119}$ Nitrogen-13 is produced by either the ${ }^{12} \mathrm{C}(\mathrm{d}, \mathrm{n})^{13} \mathrm{~N}$ or the ${ }^{14} \mathrm{~N}(\mathrm{n}, 2 \mathrm{n})^{13} \mathrm{~N}$ reaction. ${ }^{120-122}$ Sulfur-35 is usually produced by the ${ }^{34} \mathrm{~S}(\mathrm{n}, \gamma)^{35} \mathrm{~S}$ or the ${ }^{35} \mathrm{Cl}(\mathrm{n}, \mathrm{p})^{35} \mathrm{~S}$ process. ${ }^{123,124}$ The $\mathrm{HAC}$ of recoil sulfur-38 pruduced by the ${ }^{40} \mathrm{Ar}(\mathrm{p}, 3 \mathrm{p})^{38} \mathrm{~S}$ process is yet another example ${ }^{125}$ and, like ${ }^{3} \mathrm{He}(\mathrm{n}, \mathrm{p})^{3} \mathrm{~T}$ activation, permits use of an inert-gas nuclear target in gas-phase studies. The ${ }^{32} \mathrm{~S}(\mathrm{n}, \mathrm{p})^{32} \mathrm{P}$, ${ }^{35} \mathrm{Cl}(\mathrm{n}, \alpha)^{32} \mathrm{P}$, and ${ }^{31} \mathrm{P}(\mathrm{n}, \gamma)^{32} \mathrm{P}$ reactions have been used for the production of phosphorous-32. $126-129$

\section{Fission-activated HAC}

Uranium and other fission processes have been utilized in HAC studies. The HAC reactions of fission products, coupled with fast separation techniques including gas chromatography, have also been used to study the properties of fission products. ${ }^{130}$ Various studies of iodine and bromine fission isotopes ${ }^{131-135}$ as well as other elements ${ }^{136-142}$ have been reported. 


\section{Other HAC activation processes}

Activation by isomeric transition, primarily of the bromine isotopes, was summarized earlier in this review. In addition, beta decay ${ }^{143-149}$ as well as positron and electron-capture ${ }^{150-153}$ HAC activation processes have been investigated. The dissociation patterns ${ }^{154}$ and the chemistry related to the preparation ${ }^{155}$ of labelled compounds have been discussed. Lastly, positronium and muonium are among the other HAC studies that have been reported. ${ }^{156-158}$

\section{References}

1. L. Szilard, T.A. Chalmers, Nature, 134 (1934) 462.

2. K. Roessler, H.J. Jung, B. Nebeling, Adv. Space Res., 4 (1984) 83.

3. K. RoEssler, Eur. Space Agency, (Spec. Publ.) ESA SP, 1985, p. 175.

4. T. ANDERSEN, Chemical Effects of Nuclear Transformations in Inorganic Systems, North-Holland Publ. Co., Amsterdam, 1979, p. 403.

5. K. Yoshihara, T. SEKINE, Nippon Kagaku Kaishi, 11 (1984) 1873.

6. A. Mryakawa, T. SekINE, Kakuriken Kenkyu Hokoku, 19 (1986) 233.

7. K. Yoshihara, G.K. Wolf, F. Baumgaertner, Radiochim. Acta, 21 (1974) 96.

8. T. IDO, R. Iwata, Studies in Physical and Theoretical Chemistry, 31: Hot Atom Chemistry, Elsevier, Amsterdam, 1984, p. 417.

9. E. Lebowtz, P. RichardS, J. Baranosky, Int. J. Appl. Radiat. Isotopes, 23 (1972) 392.

10. T. NozakI, Stud. Phys. Theor. Chem., 31 (Hot Atom Chem), (1984) 404.

11. G.A. BRINKMAN, Int. J. Appl. Radiat. Isotopes, 33 (1982) 525.

12. T. Tominaga, E. Tachikawa, Modern Hot Atom Chemistry and Its Applications, Inorganic Chemistry Concepts 5, Springer-Verlag, Berlin, 1981.

13. D.S. URCH, MTP Int. Rev. Sci. : Inorg. Chem., Ser. 2,8 (1975) 49.

14. E.P. RACK, Radiochim. Acta, 28 (1981) 221.

15. D.S. URCH, Radiochim. Acta, 28 (1981) 183.

16. G. Harbortte, A.G. MADDOCK, (Eds.), Chemical Effects of Nuclear Transformations in Inorganic Systems, North-Holland Publ. Co., Amsterdam, 1979.

17. T. Matsuura, (Ed.), Studies in Physical and Theoretical Chemistry, 31: Hot Atom Chemistry, Elsevier, Amsterdan, 1984.

18. J.B. Evans, J.E. Willard, J. Am. Chem. Soc., 78 (1956) 2908.

19. T. Tamai, S. Nishikawa, Y. Tanaka, T. Nakamura, H. Takemi, Ann. Rep. Res. React. Inst., Kyoto Univ., 19 (1986) 124.

20. H. Seiler, M. Seiler, Helv. Chim. Acta, 50 (1967) 2477.

21. N. Nesmeyanov, A. Babeshkin, N. Koseva. Bekker, V. Lebedev, Chem. Effects Nucl. Transformations. Proc. Symp., Vienna, Vol. 2, 1964, p. 419.

22. T.A. CARLSON, Chemical Effects of Nuclear Transformations in Inorganic Systems, North-Holland Publ. Co., Amsterdam, 1979, p. 13.

23. A.A. Gordus, C. Hsiung, J. Chem. Phys., 36 (1962) 954.

24. C. Hsiung, A. Gordus, Chem. Effects Nucl. Transformations. Proc. Symp., Vienna, 1064, Vol. 2, 1965 , p. 461.

25. Z. Alfassi, S. AMIEL, Ist. J. Chem., 7 (1969) 347.

26. P.J. Estrup, R. Wolfgang, J. Am. Chem. Soc., 82 (1960) 2665.

27. C. Hsiung, A.A. Gordus, J. Am. Chem. Soc., 82 (1964) 2782. 
28. D.J. Malcolme-Lawes, Radiochim. Acta, 193 (1973) 113.

29. B. Shizgal, J. Chem. Phys., 72 (1980) 3143.

30. B. Shizgal, J. Chem. Phys., 72 (1980) 3156.

31. B. Shizgal, J. Chem. Phys., 74 (1981) 1401.

32. J. KeIZer, J. Chem. Phys., 58 (1973) 4524.

33. B. Shizgal, J. Chem. Phys., 69 (1978) 5355.

34. L.M. RAFF, J. Chem. Phys., 60 (1974) 2220.

35. A. Temkin, Chem. Phys., 99 (1985) 223.

36. D.R. WILEs, W.H. Wong, Can. J. Chem., 45 (1967) 1813.

37. V.C. ANSELMo, J. Inorg. Nucl. Chem., 35 (1973) 1069.

38. E.P. RACK, A.A. Gordus, J. Chem. Phys., 36 (1962) 287.

39. R.L. Williams, F.S. Rowland, J. Phys. Chem., 76 (1972) 3509.

40. M. Knickelbein, Z. XUE, J. RoOt. J. Phys. Chem., 88 (1984) 2017.

41. G.A. BRinkman, J. Viser, L. Lindner, Radiochim. Acta, 26 (1979) 77.

42. A. VAN DUlmen, A. ATEN, Radiochim. Acta, 15 (1971) 34.

43. R. Manning, J. Root, J. Phys. Chem., 81 (1977) 2576.

44. G.A. BRINKMAN, P. VeRnooys, Radiochem. Radioanal. Lett., 49 (1981) 95.

45. G.A. BrinkMAN, J. VISSER, Radiochim. Acta, 27 (1980) 91.

46. C.M. WA, F.S. Rowland, J. Am. Chem. Soc., 90 (1968) 3638.

47. C.M. WAI, F.S. Rowland, J. Am. Chem. Soc., 91 (1969) 1053.

48. L. Spicer, R. Wolfgang, J. Phys. Chem., 50 (1969) 3466.

49. N. Chandrasekhar, B.S.M. Rao, Radiochim. Acta, 46 (1989) 25.

50. S. Kontis, D. Maicolme-LAWES, D. UrCH, Radiochim. Acta, 24 (1977) 87.

51. A.S. AGRAWAL, B.S.M. RAO, Radiochim. Acta, 33 (1983) 81.

52. N.J. Blair, E.P. Rack, J. Chem. Phys., 48 (1968) 4085.

53. A.N. Nesmeynanov, E.S. Filatov, Radiokhimiya, 3 (1961) 601.

54. M. YAGI, K. KoNDO, Kakuriken Kenkyu Hokoku (Tohoku Daigaku), 9 (1976) 150.

55. J.J. Frost, S.M. MoERLEIN, M.J. Welch, J. Am. Chem. Soc., 103 (1981) 4337.

56. S. Mishra, N. Singh, Proc. Nucl. Chem. Radiochem. Symp. 1981, Dep. At. Energy (India), Bombay, 1983, p. 188.

57. L. Opelanio-Buencamino, F. El-Amri, W. Grauer, E. Rack, Radiochim. Acta, 37 (1984) 191.

58. J.J. Frost, S.M. Moeriein, M.J. Werch, J. Am. Chem. Soc., 103 (1981) 4332.

59. A.J. Cole, M.D. MiA, G.E. MiLleR, P.F.D. SHAw, Radiochim. Acta, 6 (1966) 150.

60. J.B. NichOlas, M. YoONG, B.P. RACK, Radiochim. Acta, 19 (1973) 124.

61. J.G. KuhrY, C.H. Hertz, J.M. PAulus, Radiochim. Acta, 20 (1973) 55.

62. M. Berg, A. Loventhal, D. Adleman, W. Grauer, E.Rack, J. Phys. Chem., 81 (1977) 837.

63. P. Geissler, J. Willard, J. Chem. Phys., 67 (1963) 1675.

64. D.D. Wilkey, J.F. Brensike, J.E. Willard, J. Phys. Chem., 71 (1967) 3580.

65. E. TACHIKAWA, T. MATSUURA, Kazaku Sosetsu, 23 (1979) 130.

66. L.D. SPICER, A. SUIDA, Radiochim. Acta, 18 (1972) 16.

67. D.J. Stevens, L.D. SPicer, J. Chem. Phys., 64 (1976) 4798.

68. E. Tachikawa, K. Numakura, Bull, Chem. Soc. Jap., 47 (1974) 2749.

69. G. BOYD, Q. LARSON, J. Am. Chem. Soc., 91,17 (1969) 4639.

70. J.JACH, G. HARBOTtLE, Trans. Faraday Soc., 54 (1958) 520.

71. Y. LiN, D. WiLes, Radiochim. Acta, 13 (1970) 43.

72. A.G. MADDOCK, M. Del VAL CoB, Trans. Faraday Soc., 55 (1959) 1709.

73. H. Mueller, P. Obergfell, I. Hagenlocher, J. Phys. Chem., 90 (1986) 3418.

74. E. Gardner, M. Gravenor, R. Harding, J. Raynor, R. Autchakt, Radiochim. Acta, 13 (1970) 100.

75. S. Mishra, R. Sharma, R. Tripathi, Indian J. Chem. Sect. A, 22A (1983) 790.

76. V. Dedgaonkar, R. LokHande, M. Chaudhari, Radiochim. Acta, 27 (1980) 101.

77. R.N. SINGH, B.M. SHUKIA, Radiochim. Acta, 27 (1980) 11. 


\section{A.A. GORDUS: HOT-ATOM CHEMISTRY}

78. S. Mishra, R Sharma, Radiat. Phys. Chem., 28 (1986) 387.

79. L. Antunes, A. Beumo, J. Radioanal. Nucl. Chem., 109 (1987) 169.

80. M. MOHAN, R.M. IYER, Radiochem. Radioanal. Lett., 8 (1971) 1.

81. J. Machado, R. Machado, J. Vargas, Chem. Effects Nucl. Transformations. Proc. Symp., Vienna, 1964, Vol. 2, 1965 p. 195.

82. M.I. StAmoul, J. Radioanal. Nucl. Chem., 102 (1986) 413.

83. Y. SaKai, H. Nishioj, T. Tommaga, Radiochim. Acta, 36 (1984) 181.

84. M. SAKanOUE, K. EnDa, Radiochem. Radioanal. Lett., 4 (1970) 99.

85. S. Vetukovic, S. Mrenkovec, M. Ratkovec, Chem. Effects Nucl. Transformations. Proc. Symp., Vienna, 1964 Vol. 2, 1965 p. 267.

86. R. ACKERHALt, G. HaRbottle, Radiochim. Acta, 17 (1972) 126.

87. E. Lazzarini, A.L. Fantola-LazZarinI, J. Inorg. Nucl. Chem., 36 (1974) 263.

88. F. Lancas, K. Collins, C. Collins, Radiochim. Acta, 38 (1985) 189.

89. K. Kishore, K VenKateswarlu, Radiochem. Radioanal. Lett., 13 (1973) 17.

90. H. JakubineK, S. SRintvasan, D. Wiles, Can. J. Chem., 49 (1971) 2175.

91. P.N. DIMOTAKIS, B.P. PAPADOPOULOS, Radiochem. Radioanal. Lett., 42 (1980) 159.

92. E. Dieffaliah, M. Ashy, R. Mahfouz, J. Radioanal. Nucl. Chem. Letters, 93 (1985) 89.

93. D.S. URCH, Radiochem. Radioanal. Lett., 25 (1976) 273.

94. T. COstea, M. Constanta, J. Inorg. Nucl. Chem., 28 (1966) 2777.

95. M. CASTIGLIONI, P. VolPE, Radiochim. Acta, 34 (1983) 165.

96. S. Daniel, Y. Tang, J. Chem. Phys., 73 (1969) 4378.

97. A. Koutr, J.M. Paulus, Radiochim. Acta, 40 (1986) 119.

98. T. Tominaga, A. Hosaka, F.S. Rowland, J. Phys. Chem., 73 (1969) 465.

99. J. SChroeder, N. Monroe, J. GARLAND, J. Phys. Chem., 73 (1969) 1252.

100. K. OOHASHI, N. MORIKAWA, Radioisotopes, 33 (1984) 601.

101. K. Mahan, J. Garland, J. Phys. Chem., 75 (1971) 1031.

102. D.J. Malcolme-LAWES, D.S. URCH, M.J. WeLCH, Radiochim. Acta, 6 (1966) 184.

103. C. MANTESCU, T. CosteA, Chemical Effects of Nuclear Transformations in Inorganic Systems, North-Holland Publ. Co., Amsterdam, 1979, p. 393.

104. R. Hall, D.J. Malcolme-Lawes, J. Chem. Soc., Faraday Trans. 1, 70 (1974) 648.

105. J. HAWKE, A. SUEDA, C. LuKEY, J. Chem. Soc. (London) Chem. Commun., 7 (1986) 499.

106. N.C. Hón, J.M. PauLus, Radiochem. Rađioanal. Lett., 23 (1975) 85.

107. E. LeE, G. Miller, F. Rowland, J. Am. Chem. Soc., 87 (1965) 190.

108. J.G. LO, J.CH. NiEH, J. Radioanal. Nucl. Chem., 97 (1986) 237.

109. Y. Aratone, E. Tachikawa, T. Mryazaki, S. Nagaya, Y. Fujttani, K. Fuekt, J. Phys. Chem., 87 (1983) 1201.

110. Y.N. Tang, F.S. Rowland, J. Phys. Chem., 72 (1968) 707.

111. D. URCH, M. WeLCH, R. ARTHY, Trans. Faraday Soc., 66 (1970) 1642.

112. T. SmaIh, F.S. Rowland, J. Phys. Chem., 72 (1968) 1845.

113. J. Root, F. Rowland, J. Chem. Phys., 38 (1963) 2030.

114. J. Roor, J. Phys. Chem., 73 (1969) 3174.

115. A. Rosenberg, R. Wolfgang, J. Phys. Chem., 41 (1964) 2159.

116. G. STOBCKLIN, Ber. Kernfoschungsanlage Juelich No. 228, 1965, p. 98.

117. R. FerRieri, A. Wolf, Y. TANG, J. Am. Chem. Soc., 16 (1983) 5428.

118. B. Nebeling, K. Roessler, G. Stoecklin, Radiochim. Acta, 38 (1985) 15.

119. J. Vaughan, V. Lieu, J. Phys. Chem., 68 (1964) 2497.

120. M. WelCh, J. Lifton, J. Am. Chem. Soc., 93 (1971) 3385.

121. Y. SEnsui, K. Tumora, T. MatsuUra, Radiochim. Acta, 35 (1984) 37.

122. V. KLIMENT, R SANDRIK, Radiochim. Acta, 30 (1982) 21.

123. L. Kremer, L. Spicer, J. Am. Chem. Soc,, 97 (1975) 5021.

124. E. LeE, Y. Tang, F. Rowland, J. Phys. Chem., 68 (1964) 318.

125. L. LINDNER, G.A. BrinKMAN, J.T. VEENBOER, Radiochim. Acta, 27 (1980) 95. 
126. P. Bebesel, C. TurCanu, Rev. Roumaine Chim., 11 (1966) 891.

127. G.W.A. NEWTON, Chemical Effects of Nuclear Transformations in Inorganic Systems, NorthHolland Publ. Co., Amsterdam, 1979, p. 343.

128. G.P. GenNaro, Y.N. TANG, J. Inorg. Nucl. Chem., 35 (1973) 3087.

129. A. Henglein, H. Drawe, D. Perner, Radiochim. Acta, 2 (1963) 19.

130. I. Fumtwara, H. Moriyama, E. Tachikawa, Studies in Physical and Theoretical Chemistry, 31: Hot Arom Chemistry, Elsevier, Amsterdam, 1984, p. 480.

131. D. ORMOND, F.S. ROWLAND, J. Am. Chem. Soc., 83 (1961) 1006.

132. M.D. SILBERT, R.H. Tomlinson, Radiochim. Acta, S (1966) 217.

133. H.O. DenschlaG, A.A. Gordus, Fresenius Z. Anal. Chem., 226 (1967) 62.

134. M. KiKUCH, L. CHURCH, Rađiochim. Acta, 20 (1973) 81.

135. N. LAVI, J. Radioanal. Chem., 20 (1974) 41.

136. F. BAUMgaertNeR, P. Reichold, Z. Naturforsch., 16a (1961) 374.

137. F. BAUMGaERTNER, A. SCHOEN, Radiochim. Acta, 3 (1964) 141.

138. U. Zarin, G. HarbottLe, J. Inorg. Nucl. Chem., 15 (1966) 925.

139. J. Biachot, L. Carraz. P. Cavaluini, A. Gadelle, A. Moussa, Phys. Chem. Fission, Proc. IAeA Symp., 2nd, 1969, p. 803.

140. H. MEINHOLD, R. REICHOLD, Radiochim. Acta, 11 (1969) 175.

141. W. BOEGL, K. BAECHMANN, Radiochem. Radioanal, Lett., 17 (1974) 239.

142. H. Morryama, I. Fuhiwara, T. Nishi, Radiochim. Acta, 30 (1982) 79.

143. H. MorTyama, 1. Fuhiwara, Radiochim. Acta, 34 (1983) 117.

144. V. Mikulaj, P. Rajec, V. Faberova, Chem. Zvesti, 28 (1974) 37.

145. G. Perlov, M. Perlow, Chem. Effects Nucl. Transformations. Proc. Symp., Vienna, 1964, Vol. 2, 1965, p. 443.

146. D. Tenorio, S. Bulbuliar, J.P. AdLoff, Radiochem. Radioanal. Lett., 24 (1976) 61.

147. A. HaLPERN, A. SoKolowsKA, J. Inorg. Nucl. Chem., 27 (1965) 1893.

148. L.M. GRACHEVA, V.D. NeFEDOV, S.A. GrACHEV, Radiokhimiya, 9 (1967) 738.

149. T. ShiokawA, Hot At. Chem. Status Rep. Proc. Panel 1974, IAFA, Vienna, 1975, p. 241.

150. T. OMORI, SHWA-Chi Wu, K. TsurumakI, T. SHiokaWA, Kakuriken Kenkyu Hokoku, 3 (1970) 123.

151. T. SotobayashI, T. SuZuKI, T. Nosa, Bull. Chem. Soc. Japan, 44 (1971) 1711.

152. J.J. SChleiffer, G. Certout, Radiochim. Acta, 20 (1973) 59.

153. S. MoERLEIN, M. WeLCH, A. WOLF, J. Am. Chem. Soc., 105 (1983) 5418.

154. S. WEXLER, Chem. Effects Nucl. Transform. Proc. Symp., Prague, 1960, p. 115.

155. R.A. FeRrierI, A.P. Wolf, Radiochim. Acta, 34 (1983) 69.

156. L. Bartal, H. Ache, Radiochim. Acta, 19 (1973) 49.

157. J. Brewer, D. Fleming, Proc. Symp. Pract. Appl. Accel. 1973, (1974) p. 235.

158. D. Fleming, Rađiat. Phys. Chem., 28 (1986) 115. 